\section{HYPERPROLACTINAEMIA ASSOCIATION WITH LUPUS NEPHRITIS DISEASE ACTIVITY}

${ }^{1}$ MS Mohamed Said*, 'WA Wan Zaidi, ${ }^{1}$ WY Kong, ${ }^{2}$ A Abd Wahab, ${ }^{2} \mathrm{H}$ Othman, ${ }^{1} \mathrm{~N}$ Abd Wahab, ${ }^{3} \mathrm{~A}$ Mohd Tamil. ${ }^{1}$ Universiti Kebangsaan Malaysia Medical Centre, Medical, Kuala Lumpur, Malaysia; ${ }^{2}$ Universiti Kebangsaan Malaysia Medical Centre, Microbiology and Immunology, Kuala Lumpur, Malaysia; ${ }^{3}$ Universiti Kebangsaan Malaysia Medical Centre, Community Health, Kuala Lumpur, Malaysia

\subsection{6/lupus-2017-000215.297}

Background and aims Prolactin has been found to be associated with immune regulation in SLE. The aim of this study is to determine the correlation between high prolactin level in comparison with IL - 6 with lupus nephritis disease activity in UKMMC.

Methods In this cross-sectional study, the analysis was conducted in SLE patients who attended Nephrology clinic in UKMMC from August 2015 till February 2016

Results Out of 43 patients with lupus nephritis, $27.9 \%$ of the patients had raised serum prolactin. The median of serum prolactin level at $0 \mathrm{~min}$ was $19.91 \mathrm{ng} / \mathrm{ml}$ (IQR: 15.95-22.65 ) for active lupus nephritis that was significantly higher as compared to the median of serum prolactin level $14.34 \mathrm{ng} / \mathrm{ml}$ (IQR: 11.09-18.70) for patients in remission $(p=0.014)$. The serum prolactin level was positively correlated to SLEDAI $\left(\right.$ rho $\left._{\mathrm{s}}: 0.449, \mathrm{p}=0.003\right)$ and UPCI level in lupus nephritis patients $\left(\mathrm{rho}_{\mathrm{s}}\right.$ : 0.241, $\left.\mathrm{p}=0.032\right)$. Assessment of serum IL-6 levels found that the active lupus nephritis patients were having a higher median level of $65.91 \mathrm{pg} / \mathrm{ml}$ (21.96-146.14) compared to in remission level of $15.84 \mathrm{pg} / \mathrm{ml}$ (IQR: 8.38-92.84), $(\mathrm{p}=0.039)$. ROC curve analysis of serum prolactin $0 \mathrm{~min}$ and serum prolactin $30 \mathrm{~min}$ and IL-6 level for prediction of SLE diseases activity provide the cutoff value of serum prolactin $0 \mathrm{~min}$ was $14.63 \mathrm{ng} / \mathrm{ml}$ with sensitivity $91.7 \%$ and specificity $58.1 \%$ and AUC of $0.74(\mathrm{p}=0.015)$.

Conclusions Baseline fasting serum prolactin level was found to be a sensitive biomarker for evaluation of lupus nephritis disease activity.

\section{IDENTIFICATION OF PLASMACYTOID DENDRITIC CELL INDUCED PROTEINS AS POTENTIAL BIOMARKERS FOR JNJ-56022473}

${ }^{1} \mathrm{~K}$ Monaghan*, ${ }^{2} \mathrm{~B}$ Linggi, ${ }^{1} \mathrm{M} \mathrm{Ng},{ }^{1} \mathrm{TY}$ Tai, ${ }^{3} \mathrm{~J} J o r d a n,{ }^{3} \mathrm{M}$ Cesaroni, ${ }^{3} \mathrm{~J}$ Benson, ${ }^{1} \mathrm{C}$ Tarlinton, ${ }^{4} \mathrm{E}$ Morand, ${ }^{4} \mathrm{~A}$ Hoi, ${ }^{1} \mathrm{~N}$ Wilson. ${ }^{1} \mathrm{CSL}$ Limited, Research, Melbourne, Australia; ${ }^{2} J a n s s e n$, Research and Development LLC, San Diego, USA; ${ }^{3}$ Janssen, Research and Development LLC, Springhouse, USA; ${ }^{4}$ Monash University, Department of Medicine, Melbourne, Australia

\subsection{6/lupus-2017-000215.298}

Background and aims Plasmacytoid dendritic cells (pDC) are potent producers of IFN $\alpha$, we investigated what additional soluble factors are produced by $\mathrm{pDCs}$ and the effect of pDC depletion with JNJ-56022473 (JNJ-473), a novel antibody against CD123. We then investigated which of these factors are elevated in SLE patient sera to determine potential biomarkers.

Methods pDCs were isolated from healthy donor (HD) PBMC $(n=6)$ which were stimulated with CpGc (TLR9) and imiquimod (TLR7) then analysed by RNAseq. PBMC from SLE and HD were also treated with isotype or JNJ-473 before stimulation with CpGc for 24 hour. Culture supernatant, SLE $(n=33)$ and HD sera $(n=34)$ was analysed by bead-based multiplex assay (Myriad U.S.A).
Results TLR9 and TLR7-agonism induced the regulation of thousands of genes, many of which were different IFN $\alpha$-subtypes. Transcripts of many other secreted proteins such as MCP-2/CCL8, IP-10/CXCL10, ITAC/CXCL11 and MIP-3ß/ CCL19 were also upregulated. Proteins of these were also found to be significantly increased in SLE sera compared to HD.

Depleting pDCs with JNJ-473 in SLE and HD PBMC cultures reduced production of many $\mathrm{CpGc}$-induced proteins including IFN $\alpha$, MCP-2/CCL8, IP-10/CXCL10, ITAC/CXCL11 and MIP-3ß/CCL19. RNAseq of SLE PBMC treated with JNJ473 before $\mathrm{CpGc}$ stimulation, confirmed significantly decreased expression of MCP-2/CCL8, IP-10/CXCL10 and ITAC/CXCL11.

Conclusions We found that pDC depletion with JNJ-473 was able to prevent TLR9-induced production of IFN $\alpha$ and various other soluble proteins which are elevated in the sera of SLE patients. We propose that these soluble factors could be useful biomarkers to determine the effectiveness of $\mathrm{pDC}$ depletion and the modulation of IFN in SLE.

\section{DIFFERENTIAL ABUNDANCE OF MIRNAS CIRCULATING IN PERIPHERAL BLOOD OF PATIENTS WITH CLASS IV LUPUS NEPHRITIS}

${ }^{1} E$ Navarro, ${ }^{2} \mathrm{R}$ Navarro, ${ }^{1} \mathrm{~L}$ Pacheco, ${ }^{3} \mathrm{H}$ Lorenzi, ${ }^{1} \mathrm{Y}$ Diaz-Olmos, ${ }^{1} \mathrm{P}$ Espana-Puccini, ${ }^{4} \mathrm{~A}$ Iglesias, ${ }^{5} \mathrm{E}$ Egea, ${ }^{1} \mathrm{D}$ Haehn, ${ }^{1} \mathrm{H}$ Gonzales, ${ }^{5} \mathrm{G}$ Garavito, ${ }^{6} \mathrm{G}$ Aroca* ${ }^{1}$ Universidad Simon Bolivar, Facultad de Ciencias Basicas Biomedicas, Barranquilla, Colombia; ${ }^{2}$ Universidad Cooperativa de Colombia, Facultad de Medicina, Santa Marta, Colombia; ${ }^{3}$ Craig Venter Institute, Infectious Diseases Department, Maryland, USA; ${ }^{4}$ Universidad Nacional de Colombia, Facultad de Ciencias de la Salud, Bogota, Colombia; ${ }^{5}$ Universidad del Norte, Division Ciencias de la Salud, Barranquilla, Colombia; ${ }^{6}$ Clinica de la Costa, Unidad de Nefrologia, Barranquilla, Colombia

\subsection{6/lupus-2017-000215.299}

Background and aims Among the organs involved in-the SLE, we can highlight the renal damage, as the largest contributor to mortality in SLE patients is estimated that nearly-50\% of SLE develop kidney disease in the first years of diagnosis. Class-IV lupus-nephritis (LN-IV) is the class of lupus nephritis most common in Colombian-patients with systemic-lupuserythematosus. MicroRNAs are important molecules involved in the pathogenesis of LN. The aim of this study was to evaluate the relative abundance of circulating microRNAs in peripheral-blood of Colombian-patients with LN class IV.

Methods an observational case-control, cross-sectional. Patientsdiagnosis by biopsy class IV lupus-nephritis was compared with patients without nephritis, and healthy-individuals was raised. These were extracted venous blood, which total RNA, which was subsequently sequenced. it was Compared Against the miRBase and Ensembl database. Differential gene expression analysis was Carried Out with edgeR and Functional analysis functional analysis was done with DIANA-miRPath. Was used as variables of selection Fold-Change $(\geq 2$ o $\leq-2)$ and False-Discovery-Rate (0.05).

Results We identified 24 circulating microRNAs with diference abundace that LNN or CTL, two of these microRNAs miR107-3p and miR375-3p are described for first time to lupus nephritis.

Conclusions This changes in the abundace of miRNA, it implies alterations in the miRNAs-mRNA regulatory network in the pathogenesis of $\mathrm{LN}$ preceding the clinical onset of the disease. The findings thus contribute to understanding the 
disease process and are likely to pave the way towards identifying disease-biomarkers for early-diagnosis of LN.

\section{PREDICTORS OF EARLY RESPONSE TO RITUXIMAB IN SYSTEMIC LUPUS ERYTHEMATOSUS (SLE): RESULTS FROM THE BRITISH ISLES LUPUS ASSESSMENT GROUP BIOLOGICS REGISTER (BILAG-BR)}

${ }^{1,2} \mathrm{~S}$ Nesbit*, ${ }^{2} \mathrm{E}$ Sutton, ${ }^{2} \mathrm{E}$ McCarthy, ${ }^{2} \mathrm{~J}$ Reynolds, ${ }^{2} \mathrm{~B}$ Parker, ${ }^{1} \mathrm{E}$ Morand, ${ }^{2} \mathrm{BILAG}-$ BR Consortium, ${ }^{2}$ Bruce. ' School of Clinical Sciences at Monash Health, Monash University, Melbourne, Australia; ${ }^{2}$ Centre for Musculoskeletal Research, University of Manchester, Manchester, UK

\subsection{6/lupus-2017-000215.300}

Background and aims The anti-CD20 agent rituximab (RTX) is generally reserved for the treatment of refractory SLE. Whist response is variable no clear predictors of early response have been confirmed. We aimed to explore factors that predict early response to RTX in a nationwide cohort of patients receiving their first RTX course.

Methods The BILAG-BR has recruited patients with refractory SLE starting RTX in the UK since Sept 2010. For this analysis we included patients who received RTX up to November 2015, had active disease at baseline, and had disease activity indices reported at baseline and 6 months. Response was defined as improvement of all active BILAG 2004 systems with no worsening in other systems or SLEDAI-2K; and no increase in glucocorticoid dose at 6 months.

Results In 197 patients $(90.36 \%$ females) 99 (50.8\%) responded at 6 months. In a multivariable model with imputation for missing variables, concomitant IV cyclophosphamide and higher baseline oral glucocorticoid dose were associated with better response. A higher baseline global BILAG-2004 score was associated with lower rates of response (Table 1).

Conclusions Early response to RTX in refractory SLE was associated with use of concomitant cyclophosphamide, higher glucocorticoid doses and lower baseline disease activity. Serology and demographic factors did not predict response. Understanding how concomitant therapy improves longer-term responses and identifying novel biomarkers of response will improve patient selection and overall outcomes for patients receiving this therapy.

Abstract 300 Table 1 Multivariable imputed model after backwards stepwise regression

\begin{tabular}{llll}
\hline Baseline factor & Odds ratio & $95 \% \mathrm{Cl}$ & $\mathbf{p}$ \\
\hline $\begin{array}{l}\text { Concomitant } \\
\text { cyclophosphamide }\end{array}$ & 4.50 & $(1.77,11.43)$ & 0.002 \\
$\begin{array}{l}\text { Global BILAG at } \\
\text { baseline }\end{array}$ & 0.96 & $(0.93,0.99)$ & 0.007 \\
$\begin{array}{l}\text { Baseline oral } \\
\text { steroid dose }\end{array}$ & $1.03^{*}$ & $(1.00,1.06)$ & 0.033 \\
\hline
\end{tabular}

*per $1 \mathrm{mg}$ increase in daily oral steroid dose. For every $10 \mathrm{mg}$ daily increase, OR 1.30

\section{EVIDENCE FOR A PRO-INFLAMMATORY AS WELL AS PROTECTIVE ROLE FOR IL-17A IN PATIENTS WITH SYSTEMIC LUPUS ERYTHEMATOSUS}

${ }^{1} \mathrm{~W}$ Raymond*, ${ }^{2} \mathrm{G}$ Ostli-Eilertsen, ${ }^{1} \mathrm{~S}$ Griffiths, ${ }^{1,2} \mathrm{~J}$ Nossent. ${ }^{1}$ The University of Western Australia, School of Medicine- Rheumatology section, Perth, Australia; ${ }^{2}$ Arctic University Norway, Dept Internal Medicine - Bone and Joint group, Tromso, Norway

\subsection{6/lupus-2017-000215.301}

Background and aims The successful application of IL-17 inhibitors in a number of chronic inflammatory diseases has increased interest for the role of IL-17 in other conditions. We investigated the clinical associations for the predominant family member IL-17A in patients with Systemic Lupus Erythematosus (SLE).

Methods Cross-sectional study of SLE patients $(n=102$, age 49, $86 \%$ female) recruited from a regional registry. IL-17A levels were determined by immunoassay, disease activity by SLEDAI-2K and cumulative damage by SLICC-DI scores. Nonparametric techniques were used to examine the association between IL-17A and disease activity, autoantibody profiles and damage development in SLE patients and for comparisons with healthy controls $(n=31)$.

Results SLE patients had higher IgG levels, lower T cell and B cell counts, but median IL-17A levels did not differ from controls (28.4 vs. $28.4 \mathrm{pg} / \mathrm{ml}, \mathrm{p}=0.9)$.

In SLE patients, IL-17A levels did not correlate with SLEDAI-2K or SDI, but were inversely related with systolic blood pressure $(\mathrm{r}=-0.31, \mathrm{p}=0.02)$, years smoking $(\mathrm{r}=-0.23$, $\mathrm{p}<0.001)$, cumulative heart $(\mathrm{r}=-0.24, \mathrm{p}=0.03)$ and malignancy damage $(\mathrm{r}=-0.18, \mathrm{p}=0.06)$.

Serological correlations for IL-17A existed with levels of IgG $(r=0.21, p=0.05)$, hs-CRP levels $(r=0.28, p<0.01)$, proteinuria $(\mathrm{r}=0.64, \mathrm{p}=0.004)$ and pre-albumen $(\mathrm{r}=-0.22$, $\mathrm{p}=0.03)$.

Longitudinal data showed only modest fluctuations in $17 \mathrm{~A}$ levels, unrelated to SLEDAI-2K

Conclusions These data indicate that while IL-17A participates in the inflammatory process in SLE, it also seems to serve a protective purpose in reducing heart disease and cancer in SLE. This dual role is consistent with experimental and clinical data and raises concerns about inhibting IL-17 in SLE patients.

\section{SERUM CYTOKINE ANALYSIS AND TRANSCRIPTIONAL BLOOD PROFILING REVEAL AN ASSOCIATION BETWEEN IL-3 AND IFN IN HUMAN SLE}

${ }^{1} \mathrm{~S}$ Oon*, ${ }^{2} \mathrm{~K}$ Monaghan, ${ }^{2} \mathrm{M} \mathrm{Ng},{ }^{3} \mathrm{~A}$ Hoi, ${ }^{3} \mathrm{E}$ Morand, ${ }^{2} \mathrm{~N}$ Wilson, ${ }^{1} \mathrm{I}$ Wicks. ${ }^{1}$ The Walter and Eliza Hall Institute of Medical Research, Inflammation, Parkville, Australia; ${ }^{2}$ CSL Limited, Pre clinical biology, Parkville, Australia; ${ }^{3}$ Monash University, Medicine, Melbourne, Australia

\subsection{6/lupus-2017-000215.302}

Background and aims IFN $\alpha$, produced by plasmacytoid dendritic cells (pDCs) is a major contributor to SLE pathogenesis. IL-3 promotes pDC survival, but its role in SLE has not been well characterised. This study investigated serum IL-3 and IFN $\alpha$ levels, and a whole blood 'IL-3 gene signature' in human SLE.

Methods Serum cytokine levels were measured by ELISA in $\mathrm{n}=42$ SLE donors from The Royal Melbourne Hospital and $n=44$ healthy donors (HD). IL-3 upregulated genes were determined by RNASeq of HD whole blood (WB) stimulated 